mens viele Normen des Besonderen Teils des Strafgesetzbuches abgehandelt werden. Dies könnte zu einer Überforderung der Studierenden führen, die sich in einem so frühen Stadium ihrer Ausbildung bereits mit derart vielen Normen befassen sollen.

Für österreichische Studierende ist das Buch sicherlich empfehlenswert. Für diejenigen in Deutschland ist es eher nicht nutzbar, denn obwohl die Methodik selbstverständlich auch für sie außerordentlich wichtig ist, so sollten sie diese doch anhand der deutschen Rechtsnormen erlernen.

Katrin Stegemann-Siebert

Staatsanwältin, Kiel

\title{
Erfolgreicher Start des Netzwerks für juristische Gleichstellungsbeauftragte
}

\section{Eva Schübel \\ Vizepräsidentin des djb; Bundesanwältin beim BGH, Karlsruhe}

Die Gleichstellungsbeauftragten werden nicht selten mit der Bemerkung konfrontiert „Ist das überhaupt gleichstellungsrelevant? “, was heißen soll: Hat die Gleichstellungsbeauftragte da eigentlich mitzureden? Möglichst nicht und wenn, dann allenfalls durch Kenntnisnahme einer verfügten Maßnahme - dies ist eine nach wie vor bei Verwaltungen und Dienststellenleitungen weit verbreitete Auffassung. Die Gleichstellungsgesetze werden nicht selten als quantité négligable betrachtet und beiseite gelassen. Dies ist allerdings nicht mehr gänzlich ungefährlich. Nach einer Entscheidung des Verwaltungsgerichts Frankfurt ${ }^{1}$ stellt die fehlende Mitwirkung der Gleichstellungsbeauftragten bei den in $\$ 19$ Absatz 1 BGleiG genannten personellen, organisatorischen und sozialen Angelegenheiten einen absoluten Verfahrensfehler dar.

Eine effektive Mitwirkung beansprucht freilich - wie bei den Personaldezernenten auch - Arbeitszeit. Sieht das Gleichstellungsgesetz wie in einzelnen Bundesländern keine Entlastung der Gleichstellungsbeauftragten von den anderweitigen Dienstgeschäften vor oder nimmt diese die gesetzlich vorgesehene Möglichkeit lieber nicht in Anspruch, wird sich nicht lange nach der Wahl Frust einstellen: Wem praktisch keine Zeit für die Amtsausübung bleibt, der kann keinen Einfluss geltend machen. Eine oft geäußerte Klage bei den Brühler Gleichstellungstagen, die ich aus eigener Erfahrung bestätigen kann. Wer das Amt der Gleichstellungsbeauftragten neben einer Vollzeittätigkeit mit erledigt, kann nur bei sehr wenigen Personalentscheidungen nachhaken, weil schon der Aktenumlauf und die unbedingt erforderlichen Gespräche und Besprechungen viel Zeit beanspruchen. Nur mit einem nicht zu knapp bemessenen Freiraum kann die Gleichstellungsbeauftragte ihre Mitwirkungsrechte effektiv durchsetzen und die gesetzlich vorgesehenen Controllingaufgaben wahrnehmen - denn ablehnende Stellungnahmen zu fertigen und Einsprüche zu begründen, kostet Zeit. Dann allerdings bläst ihr meist kräftiger Wind entgegen und sie benötigt neben einem breiten Kreuz auch juristischen und moralischen Beistand von Kolleginnen. Aus dieser Erkenntnis ist bei mir die Idee entstanden, Gleichstellungsbeauftragte zu vernetzen, die wie ich Juristinnen sind.
Für die Gründung eines solchen Netzwerks ist die Zeit offensichtlich reif gewesen. Seit der Billigung des Projekts durch den djb-Bundesvorstand im November 2011 haben wir dafür schon 48 Juristinnen gewinnen können, die als Gleichstellungs- bzw. Frauenbeauftragte tätig sind oder sich hauptberuflich mit Gleichstellungsaufgaben befassen. Am 24. August 2012 fand in Berlin das Gründungstreffen mit 17 Teilnehmerinnen statt, bei dem insbesondere die Arbeitsweise und Tätigkeitsfelder des Netzwerks vereinbart worden sind. Die Mitgliedsfrauen arbeiten per djb-Internetforum zusammen und treffen sich einmal im Jahr in Berlin. Wer in das Netzwerk aufgenommen werden möchte, muss Mitglied des djb sein, weil der djb für die Kosten aufkommt, insbesondere seine Geschäftsstelle die Organisation unterstützt. Das Netzwerk dient vorrangig dem fachlichen Austausch und der kollegialen Beratung zwischen seinen Mitgliedsfrauen. Wir wollen aber auch die Stellung, Aufgaben und Rechte der Gleichstellungsbeauftragten näher beleuchten, uns zu Gleichstellungsthemen aus juristischer Sicht äußern und Stellung zu Gesetzentwürfen im Gleichstellungsrecht in Abstimmung mit der jeweils zuständigen djb-Kommission nehmen. Durch Veröffentlichungen und die Zusammenarbeit mit Netzwerken, die ähnliche Ziele verfolgen, möchten wir die Ergebnisse unserer Arbeit anderen Gleichstellungsbeauftragten bekannt machen. Daneben werden wir den Kontakt in die Politik hinein suchen. Eine Sammlung von Urteilen, die auf Gleichstellungsrecht und das Amt der Gleichstellungsbeauftragten Bezug nehmen, und eine Liste von im Gleichstellungsrecht spezialisierten Rechtsanwältinnen und Rechtsanwälten sollen die Netzwerkarbeit unterstützen.

Das erste regelmäßige Treffen des Netzwerks fand am 9. November 2012 mit 17 Kolleginnen statt. Richter am Bundesverwaltungsgericht Prof. Dr. Jan Hecker referierte über die Entscheidung des Bundesverwaltungsgerichts vom 8. April $2010^{2}$ zum Teilnahmeanspruch der Gleichstellungsbeauftragten an Führungsklausuren ihrer Dienststelle. Für die Praxis lassen sich daraus kurz zusammengefasst folgende Schlüsse ziehen: $\mathbb{2} 20$ Absatz 1 Satz 3 BGleiG, wonach der Gleichstellungsbeauftragten Gelegenheit zur aktiven Teilnahme an allen Entscheidungsprozessen zu personellen, organisatorischen

1 Beschluss v. 4.10.2011, 9 L 2202/11.F.

26 C 3.09. 
und sozialen Angelegenheiten gegeben werden soll, gilt erst, wenn sich ein Entscheidungsprozess förmlich etabliert hat. Ist eine Besprechung Teil dieses Prozesses, hat die Gleichstellungsbeauftragte Anspruch auf Teilnahme. Sie ist aber nicht zu jeder Dienstbesprechung in innerdienstlichen Angelegenheiten hinzuzuziehen, sondern nur, wenn es dabei um eine wesentliche Weichenstellung oder wesentliche Steuerung dieser Angelegenheiten geht. In der anschließenden Diskussion wurde deutlich, dass der Teilnahmeanspruch der Gleichstellungsbeauftragten trotz dieser Entscheidung des Bundesverwaltungsgerichts auch künftig nicht leicht durchzusetzen sein wird, weil wesentliche Fragen in Vorgespräche verlagert werden können oder eine beteiligungsrelevante Besprechung - wie in einer Parallelentscheidung des Verwaltungsgerichts Hamburg - als bloßer Brainstorming-Prozess deklariert wird.

Beim nächsten Netzwerktreffen Mitte 2013 wird es um die geschlechtergerechte Beurteilung und die Zielrichtung der geplanten Novellierungen der Gleichstellungsgesetze gehen. Bei Letzteren besteht die Gefahr, dass sie sich fortschrittlich geben, tatsächlich aber ein Rückschritt sein werden. Aus dem Bundesgleichstellungsgesetz sollte ein Chancengleichheitsgesetz werden. Frauenförderung gilt als überholt, Gleichstellung wird als erreicht vorausgesetzt. Gender Mainstreaming und neuer- dings Diversity sind in aller Munde. Angesichts dessen sind eine klare Bestimmung und Abgrenzung der unterschiedlichen Begriffe sowie eine Bestandsaufnahme erforderlich, um die politische Diskussion auf den Boden der Tatsachen zurückzubringen. Die Geschlechtergleichstellung ist in zentralen Bereichen unserer Gesellschaft noch nicht durchgesetzt - ein Blick in die Führungsetagen der Wirtschaft und der öffentlichen Hand genügt. Damit ist sie jedoch, wie die Richterin des Bundesverfassungsgerichts Prof. Dr. Susanne Baer in einem Interview ${ }^{3}$ ausführt, an einem Punkt angelangt, wo sie ernsthaft Wirkung zeigt und damit auch weh tun kann. Dies erklärt den heftiger werdenden Widerstand und den Versuch, die Gleichstellung von Frauen unter den Begriff Diversity zu packen. Die Hälfte der Bevölkerung ist freilich keine Chancengleichheit suchende Minderheit. Wir Juristinnen sollten daher Artitel 3 Absatz 2 GG, wonach der Staat die tatsächliche Durchsetzung der Gleichberechtigung von Frauen und Männern fördert und auf die Beseitigung bestehender Nachteile hinwirkt, in Erinnerung rufen und die Gleichstellungspolitik auf dieser Grundlage weiterzuentwickeln suchen.

3 Femina Politica 2/2012 S. 34.

\section{Deutsche Juristinnen - nun auch in Spanien vernetzt}

\author{
Katharina Miller, LL.M. \\ Abogada inscrita, Collado Mediano, Spanien
}

Für einige unter Ihnen wird es eine überraschende Neuigkeit sein, dass in Spanien viele deutsche Juristinnen leben und arbeiten. Auch gibt es hier viele spanische Juristinnen, die in irgendeiner Form mit Deutschland zu tun haben, sei es wegen eines deutschen Elternteils, sei es wegen des deutschen Lebenspartners oder aus eigenem Interesse (zum Beispiel über Erasmus) an Alemania.

Seit diesem Sommer gibt es eine Regionalgruppe Madrid in Gründung. Neben der Regionalgruppe Brüssel soll dies die zweite ausländische Gruppe des djb werden.

Am 25. Oktober 2012 trafen sich sechs deutsche und spanische Juristinnen zum ersten Mittagsstammtisch, von den Veranstalterinnen kurz almuerzo (Mittagessen) genannt. Dieses Treffen diente lediglich zum gegenseitigen Kennenlernen der sechs Anwesenden und zum Vorstellen der Ziele des djb. Für den zweiten und alle weiteren almuerzos hat das Goethe Institut Madrid freundlicherweise einen seiner Räume zur Verfügung gestellt, wo die Treffen in Ruhe und mit Menüs zu erschwinglichen Preisen stattfinden können.

Der zweite almuerzo im November hatte das für Spanien geplante Projekt „Frauen in die Aufsichtsräte” zum Gesprächsgegenstand. Denn die Projektstudie des djb „Aktionärinnen fordern Gleichberechtigung" hat viele spanische und internationale Frauenvereinigungen in Madrid überzeugt, mit der Folge, dass nun auch in Spanien ein ähnliches Projekt durchgeführt werden soll. Der Dezember-almuerzo ist aufgrund der vollen Terminkalender der Interessentinnen und Mitglieder auf den Januar verschoben worden und dem leider immer noch aktuellen Thema Gewalt gegen Frauen gewidmet, wobei Vortragende die aktuelle Präsidentin des Zonta-Clubs Madrid, Frau Inés Chamarro Storms, sein wird. Im Jahr 2013 werden sich voraussichtlich weitere vier almuerzos Themen widmen wie „Frauen in die Aufsichtsräte“, Gleichberechtigung in Spanien, der nichtehelichen „Lebensgemeinschaft in der spanischen Rechtsordnung “ sowie den Müttern der spanischen Verfassung. Dabei wird das erste Thema von Frau Eva Levy, unter anderem Gründerin der spanisch-israelischen Handelskammer und Headhunterin für Frauen in Führungspositionen, behandelt, die uns vor allem über den Vorschlag der EU-Richtlinie „zur Gewährleistung einer ausgewogeneren Vertretung von Frauen und Männern unter den nicht geschäftsführenden Direktoren/Aufsichtsratsmitgliedern börsennotierter Gesellschaften und über damit zusammenhängende Maßnahmen" informieren möchte; die beiden nächsten Komplexe werden von Interessentinnen am djb vorgetragen und für letzteres Thema werden wir die Ehre haben, eine der Mütter der spanischen Verfassung und zugleich eine der ersten Parlamentarierinnen, Frau Mercedes Moll, als Gesprächspartnerin und Vortragende in unserer Mitte empfangen zu dürfen. Weiterhin hat Frau Prof. Dr. Jutta Limbach, die Madrid sehr 